\title{
0 impacto da cirurgia de ceratectomia fotorrefrativa (PRK) e ceratomileuse assistida por excimer laser in situ (LASIK) na qualidade visual e de vida em pacientes com ametropias
}

\author{
The impact of photorefractive excimer laser keratectomy (PRK) and laser in situ \\ keratomileusis (LASIK) on visual quality and life in patients with ametropias
}

\author{
Ricardo Belfort ${ }^{1}$ \\ Mauro Campos ${ }^{2}$ \\ Marcelo Queiroz Hoexter ${ }^{3}$ \\ Rubens Belfort Jr. ${ }^{4}$ \\ Jair de Jesus Mari ${ }^{5}$
}

\begin{tabular}{l} 
RESUMO \\
\hline Objetivo: Avaliar a qualidade de vida e de visão e o estresse de pacientes \\
portadores de ametropias submetidos a procedimentos cirúrgicos. Méto- \\
dos: Estudo longitudinal observacional em que foram estudados 100 \\
pacientes; 54 usuários de óculos, 21 usuários de lentes de contato in- \\
teressados no procedimento cirúrgico e 25 controles usuários de óculos \\
ou lentes de contato, mas que não desejavam ser operados no período de \\
um ano. Os questionários aplicados foram o National Eye Institute Visual \\
Function Questionnaire (NEI-VFQ-25) de qualidade de vida e o Self \\
Reporting Questionnaire - SRQ-20 para avaliação da saúde mental. Os \\
pacientes que se submeteram à cirurgia responderam aos questionários \\
aplicados por uma observadora antes da mesma, três, seis e doze meses \\
após a intervenção. O grupo controle respondeu de forma auto-aplicada \\
no início do estudo, seis e doze meses após a primeira avaliação. Re- \\
sultados: No grupo da cirugia dos 54 pacientes que usavam óculos 39 \\
fizeram cirurgia de ceratectomia fotorrefrativa por excimer laser(PRK)e \\
15 fizeram ceratomileuse assistida por excimer laser in situ (LASIK) e dos \\
21 que usavam lentes de contato 12 fizeram cirurgia de ceratectomia \\
fotorrefrativa e nove fizeram ceratomileuse assistida por excimer laser in \\
situ (LASIK). O grupo controle esteve estável durante os 12 meses em \\
relação aos instrumentos aplicados. Três meses após a cirurgia o grupo da \\
cirurgia apresentou melhora significante da qualidade de vida e de visão \\
em relação ao pré-operatório independentemente do tipo de cirurgia \\
realizada. Um ano após a cirurgia os índices de qualidade de vida e de saúde \\
mental, foram semelhantes aos do grupo controle. O Self Reporting \\
Questionnaire - SRQ 20 mostrou diminuição significante do índice de \\
sintomas a partir dos três meses de pós-operatório. Conclusão: A quali- \\
dade de visão e de vida dos pacientes submetidos à cirurgia de correção \\
de ametropia mudou significantemente para melhor em relação ao pré- \\
operatório, com redução substancial do estresse psicológico. \\
\hline
\end{tabular}

Descritores: Ceratomileuse assistida por excimer laser in situ; Ceratectomia fotorrefrativa por excimer laser; Erros de refração/cirurgia; Miopia/cirurgia; Qualidade de vida; Estresse psicológico; Questionários

\section{INTRODUÇÃO}

Alguns autores avaliaram 45 pacientes antes da cirurgia de ceratectomia fotorrefrativa (PRK) e seis meses após pelo Cantril Self Anchoring Striving 
Scale (CSASS) e pelo questionário de sintomas psiquiátricos Symptom Checklist-90-Revised (SCL-90R) $)^{(1)}$ e concluiram que a função visual de todas as atividades da vida diária foram melhores após a cirurgia. Somente os itens dirigir durante o dia e visão para perto não tiveram diferenças estatisticamente significantes. Sobre os aspectos psicopatológicos os baixos míopes mostraram mudanças altamente significantes no pós-operatório, com melhora nos itens de hostilidade e paranóia. Para os altos míopes houve diferenças significantes na somatização, depressão, paranóia e hostilidade. Quanto ao sofrimento nos primeiros três dias após a cirurgia, 30\% o consideraram moderado e $30 \%$ pequeno. Sobre o resultado da cirurgia, $77 \%$ a julgaram muito satisfatório e $66 \%$ acharam que correspondeu à expectativa ${ }^{(1)}$.

Outros autores apresentaram os resultados do estudo das características psicológicas e demográficas de pacientes que se submeteram à cirurgia de PRK comparados a um grupo de altos míopes não detectando depressão ou ansiedade ou desvio psicológico nos pacientes que se apresentaram para cirurgia refrativa motivados pela necessidade de se livrarem dos óculos ou das lentes de contato ${ }^{(2)}$. Outro estudo mostrou que dois anos após a cirurgia de PRK, 94,3\% dos pacientes consideraram que a sua visão havia melhorado em relação ao pré-operatório ${ }^{(3)}$.

Foram avaliadas as razões pelas quais os pacientes desejavam fazer cirurgia de miopia e a sua satisfação. Para tanto desenvolveram um questionário funcional de 38 itens. As razões para se submeterem à cirurgia foram: dificuldade de usar lentes de contato e o desejo de se libertar dos óculos. Os resultados mostraram que $85,5 \%$ dos pacientes sentiram que a qualidade de suas vidas havia melhorado e $82 \%$ de acordo com a expectativa e $23,9 \%$ consideraram a cirurgia estressante ${ }^{(4)}$.

Autores estudaram a ansiedade, o estresse, a depressão e a autoestima de pacientes míopes que se apresentavam para cirurgia pelo PRK em estudo controlado ${ }^{(5)}$. Foram estudados 90 pacientes míopes e 50 míopes usuários de lentes de contato como controles. Ambos os grupos submeteram-se a dois questionários auto-aplicáveis: o General Health Questionnaire (GHQ-30) e o Hudson Index of Self-Steem (ISE) da Universidade de Chicago.

Outros autores fizeram um estudo de qualidade de vida comparando os efeitos de diferentes graus de miopia com pacientes com ceratocone ${ }^{(6)}$. Ao todo foram estudados 142 pacientes: 30 pacientes com alta miopia $-10,00 \mathrm{D}, 40$ com miopia moderada entre $-4,00$ a $-9,75 \mathrm{D}, 42$ com baixa miopia $<-4,00$ D e 30 com ceratocone. A coleta dos dados incluiu acuidade visual binocular por $\log$ MAR. A qualidade de vida foi avaliada pelo Visual Function (VF 14), e Vision Specific Quality Of Life (VQOL) e entrevistas semi-estruturadas. A única diferença significante encontrada foi na comparação entre os com alto grau de miopia com os de baixa e moderada miopia $\mathrm{p}<0,001$ assim como nos questionários VF 14 e VQOL. Os pacientes com alto grau de miopia tinham uma qualidade de vida tão pobre quanto os com ceratocone ${ }^{(6)}$.

Foi estudada a qualidade de vida de 310 pacientes com 60 anos ou mais. Todos os pacientes tiveram os seus olhos examinados e a sua acuidade visual mensurada. Havia um grupo que foi operado de catarata e outro que não realizou a cirurgia com acuidade visual de $6 / 60$ ou pior $^{(7)}$. A qualidade de vida foi avaliada pelo VF e pelo Quality Of Life (QOL). 59,6\% dos olhos operados apresentavam acuidade visual melhor que 6/18. Os resultados do VF e do QOL diminuíam quanto pior era a visão. Os autores concluíram que em Hong Kong o pósoperatório não é tão bem realizado quanto deveria.

Os objetivos deste trabalho foram avaliar o impacto de procedimento cirúrgico refrativo sobre a qualidade de visão e de vida de usuários de óculos e lentes de contato, e o estado psicológico destes pacientes antes e após a cirurgia comparando com um grupo controle que não desejava ser operado.

\section{MÉTODOS}

Foi realizado ${ }^{(8)}$ um estudo comparativo da validade de dois questionários similares o Questionário Geral de Saúde - (GHQ12) ${ }^{(9)}$ e o Questionário de Auto Avaliação Self-Reporting Questionnaire - (SRQ-20) ${ }^{(10)}$, comparando-os com uma entrevista clínica semi-estruturada. Os questionários foram aplicados em três centros de saúde da cidade de São Paulo; um deles na Brasilândia, outro no Bom Retiro e outro no Ibirapuera, que atendem populações distintas. Submeteram-se aos questionários 875 pacientes e 260 foram entrevistados. A correlação entre os dois questionários foi de $+0,72$ e os coeficientes de validação para o GHQ-12 e para o SRQ-20 foram respectivamente: sensibilidade $85 \%$ e $83 \%$; especificidade $79 \%$ e $80 \%$ e o total de falsos negativos foi $18 \%$ e $19 \%$. O questionário tem sido aplicado em vários estudos epidemiológicos no Brasil ${ }^{(11)}$.

\section{Procedimentos}

Os pacientes avaliados eram do ambulatório de cirurgia refrativa do Departamento de Oftalmologia da Escola Paulista de Medicina - UNIFESP, por demanda espontânea e usuários de óculos ou lentes de contato, que optaram pela correção cirúrgica da ametropia (grupo de intervenção) após uma explicação detalhada do médico a respeito da conveniência de se realizar a cirurgia e de seus riscos. Quanto aos critérios de inclusão os pacientes operados estavam com miopia estacionada e com exames oftalmológicos compativeis para a cirurgia. Estes pacientes respondiam ao questionário de qualidade de vida NEIVFQ $25^{(12)}$ e ao SRQ $20^{(10)}$ antes da cirurgia, 3, 6 e 12 meses após a mesma. Os controles avaliados foram voluntários usuários de óculos ou lentes de contato que não pretendiam se submeter à cirurgia no prazo de um ano. $\mathrm{O}$ grupo controle foi avaliado em três oportunidades: no tempo zero, após seis meses e após um ano. Trata-se, portanto, de um estudo observacional prospectivo. O presente estudo foi submetido e aprovado pela comissão de ética em pesquisa da UNIFESP.

\section{Análise estatística}

Para se avaliar possíveis diferenças entre os grupos para as variáveis previamente definidas usou-se o teste não paramétrico para amostras independentes de Kruskal-Wallis com- 
plementado, quando necessário, pelo teste de comparações múltiplas ${ }^{(13)}$. Para o estudo das diferenças entre dois grupos independentes usou-se o teste não paramétrico de MannWhitney. Quando a comparação para cada variável foi feita ao longo do tempo foi usado o teste não paramétrico para amostras não independentes de Friedman, complementado, quando necessário, pelo teste de comparações múltiplas. Quando esta variável foi utilizada para a comparação de três grupos o teste utilizado foi Kruskal-Wallis. Em todos os casos foi usado para nível de rejeição da hipótese de nulidade o valor menor ou igual a 0,05 (5\%). Quando a estatística calculada apresentou significância foi usado um asterisco ${ }^{*}$ ) para caracterizá-la ${ }^{(14)}$.

\section{RESULTADOS}

O grupo que se submeteu à cirurgia foi composto por 75 pacientes: 54 que usavam óculos e 21 lentes de contato. A média das idades dos pacientes que usavam óculos antes da cirurgia foi de 31,77 anos e no grupo dos que usavam lentes de contato foi 30,33 anos. Os que usavam óculos e eram do sexo masculino foram $31,5 \%$ e os que usavam lentes foram $52,4 \%$. No grupo controle a média de idade foi de 32,48 anos e $40 \%$ eram do sexo masculino. Portanto, os grupos foram homogêneos em relação ao gênero e a idade. Quanto à escolaridade as maiores porcentagens foram referentes ao curso superior completo tanto no grupo de intervenção subdividido quanto no grupo controle como mostra a tabela 1.

Com a cirurgia as ametropias diminuíram nos dois olhos operados tanto nos pacientes que usavam óculos quanto nos que usavam lentes de contato ao contrário do grupo controle que não apresentou alteração durante o ano como mostra a tabela 2. O teste de Friedman mostra que a miopia do olho direito diminuiu significantemente a partir dos três meses após a cirurgia quando os questionários foram aplicados pela segunda vez. O mesmo aconteceu com a miopia do olho esquerdo. O grupo controle se manteve estável.
Os resultados em relação à qualidade de vida e da saúde mental estão descritos na tabela 3. No grupo que usava óculos 6 meses após a cirurgia quando os questionários foram aplicados pela terceira vez houve diferença significante entre os que fizeram PRK e ceratomileuse assistida por excimer laser in situ (LASIK) (Z-2,713)*. O grupo que usava óculos e fez PRK obteve maior melhora da qualidade de vida do que o que fez LASIK nesta fase do estudo. Os que fizeram PRK tiveram média de 93,44 e os que fizeram LASIK 88,35 no resultado total do NEIVFQ. Na comparação dos escores totais do The National Eye Institute - Visual Function Questionnaire (NEI-VFQ) dos grupos PRK e LASIK e óculos e lente de contato no pré-operatório, pós-operatório de 3 meses e um ano o teste de Kruskal-Wallis não detectou diferença significante.

Em relação ao Self Reporting Questionnaire (SRQ 20) o teste de Friedman mostrou que houve diferença no grupo de pacientes que fizeram cirurgia a partir de 3 meses de pósoperatório em relação ao pré (de 4,14 para 2,76), sendo esta diferença estatisticamente significante.

Os pacientes do grupo da cirurgia tiveram a qualidade de vida e de visão melhorada após a cirurgia enquanto o grupo controle não apresentou alteração como mostra o gráfico 1 .

Os pacientes que fizeram PRK e usavam óculos e os que usavam lentes de contato apresentaram melhor qualidade de vida ao longo do ano após a cirurgia enquanto que o grupo controle foi o que menos teve alteração. Os pacientes que usavam óculos e fizeram LASIK só têm o resultado de 6 meses após a cirurgia porque houve diferença significante com o grupo que fez PRK e usava óculos.

Quanto ao SRQ 20 o grupo de intervenção apresentou melhora nos sintomas psicológicos enquanto que o grupo controle se manteve sem alteração ao longo do ano como mostra o gráfico 2.

O teste de Mann Withney que comparou os grupos que fizeram PRK e LASIK avaliou o total do questionário NEIVFQ para os pacientes que usavam óculos foi aplicado nas quatro

\begin{tabular}{|c|c|c|c|c|c|c|}
\hline \multirow{4}{*}{ Média de idade (anos) } & \multicolumn{6}{|c|}{ Intervenção } \\
\hline & \multirow{2}{*}{\multicolumn{2}{|c|}{ Óculos }} & \multirow{2}{*}{\multicolumn{2}{|c|}{$\begin{array}{c}\text { Lentes de contato } \\
30,33\end{array}$}} & \multirow{2}{*}{\multicolumn{2}{|c|}{$\begin{array}{c}\text { Controle } \\
32,48\end{array}$}} \\
\hline & & & & & & \\
\hline & Pacientes & $\%$ & Pacientes & $\%$ & Pacientes & $\%$ \\
\hline Sexo masculino & 17 & $(31,50)$ & 11 & $(52,40)$ & 10 & $(40)$ \\
\hline Sexo feminino & 37 & $(68,50)$ & 10 & $(47,62)$ & 15 & $(60)$ \\
\hline Superior completo & 22 & $(40,74)$ & 10 & $(47,62)$ & 17 & $(68)$ \\
\hline Superior incompleto & 8 & $(14,81)$ & 1 & $(4,76)$ & 1 & (4) \\
\hline Médio completo & 15 & $(27,77)$ & 8 & $(38,00)$ & 7 & $(28)$ \\
\hline Médio incompleto & 7 & $(13,00)$ & 2 & $(9,62)$ & & \\
\hline Fundamental completo & 1 & $(1,84)$ & & & & \\
\hline Fundamental incompleto & 1 & $(1,84)$ & & & & \\
\hline PRK & 39 & $(72,22)$ & 12 & $(57,14)$ & & \\
\hline LASIK & 15 & $(27,78)$ & 9 & $(42,86)$ & & \\
\hline Total & 54 & 100 & 21 & 100 & 25 & 100 \\
\hline
\end{tabular}


fases do estudo. Como pode ser verificada na tabela 3 a qualidade de vida foi superior para o grupo PRK $(93,44)$ do que o grupo LASIK $(88,35)$ após seis meses de cirurgia sendo esta diferença estatisticamente significante.

O teste de Mann Witney que avaliou o resultado total do questionário NEIVFQ também foi aplicado nos pacientes que usavam lentes de contato. O objetivo foi comparar os grupos que fizeram PRK e LASIK nos quatro momentos do estudo. Não houve diferenças significantes entre estes dois grupos (Tabela 3).

São apresentados os resultados do questionário NEIVFQ dos pacientes que usavam óculos no decorrer de um ano como mostra a tabela 4. Nesta tabela pode-se notar que houve diferença estatística significante para o quesito visão para longe no grupo que usava óculos já a partir dos 3 meses em relação ao pré-operatório quando os questionários foram aplicados pela seguinda vez. No quesito saúde mental ao longo do ano o teste de Friedman detectou uma diferença significante para o grupo que usava óculos a partir dos 3 meses do pré-operatório. O teste de Friedman avaliou o desempenho de papéis ao longo do ano. O grupo que usava óculos mostrarou diferença a partir dos 3 meses em relação ao pré-operatório. No quesito visão periférica o teste de Friedman encontrou diferença ao longo do ano no grupo que usava óculos a partir dos 6 e 12 meses quando os questionários foram aplicados pela terceira e quarta vez.

São apresentados os resultados do NEIVFQ dos pacientes que usavam lentes de contato antes da cirurgia como mostra a tabela 5 .

Esta tabela mostrou diferença estatística para os pacientes que usavam lentes de contato já a partir dos três meses de

\begin{tabular}{|c|c|c|c|c|}
\hline & \multirow[t]{2}{*}{ Pré-operatório } & \multicolumn{3}{|c|}{ Pós-operatório } \\
\hline & & 3 meses & 6 meses & 12 meses \\
\hline Óculos-OD & $-4,08$ & $0,23^{*}$ & 0,06 & $-0,15$ \\
\hline Óculos-OE & $-4,07$ & $-0,05^{\star}$ & $-0,02$ & $-0,27$ \\
\hline LC-OD & $-4,34$ & $-0,17^{*}$ & 0,25 & 0,33 \\
\hline LC-OE & $-4,46$ & $-0,17^{*}$ & 0,25 & $-0,11$ \\
\hline Controle-OD & $-2,16$ & & $-1,94$ & $-1,96$ \\
\hline Controle-OE & $-2,13$ & & $-2,02$ & $-1,88$ \\
\hline
\end{tabular}

pós-operatório no sofrimento ocular. Os pacientes que usavam lentes de contato (LC) deixaram de sofrer e ficaram mais satisfeitos. Esta tabela mostrou também que os pacientes que usavam lentes de contato tiveram melhora significante aos 12 meses (última aplicação dos questionáriuos) no quesito visão para perto em relação ao pré-operatório.

No quesito saúde mental ao longo do ano o teste de Friedman detectou que o grupo que usava LC melhorou a partir dos 3 meses na segunda aplicação dos questionários. Em relação ao desempenho de papéis ao longo do ano o grupo que usava LC mostrou diferença significante a partir dos 3 meses em relação ao pré-operatório. A tabela 6 descreve os escores de qualidade de vida no grupo controle, não havendo diferenças importantes ao longo do ano.

\section{DISCUSSÃO}

O NEIVFQ foi escolhido para este estudo por se tratar de um questionário utilizado para pacientes com diversas doenças oculares ${ }^{(11)}$. O NEIVFQ modificado foi aplicado em pacientes que se submeteram à cirurgia refrativa com excimer laser no Departamento de Oftalmologia da UNIFESP - EPM com o intuito de avaliar a qualidade de visão antes da cirurgia, três meses, seis meses e um ano após a intervenção. Trata-se de um questionário dirigido exclusivamente aos pacientes oftalmológicos, traduzido do inglês e adaptado no próprio Departamento por profissionais da área de saúde habilitados para atendimento oftalmológico. Este questionário é composto de 25 perguntas com respostas de múltipla

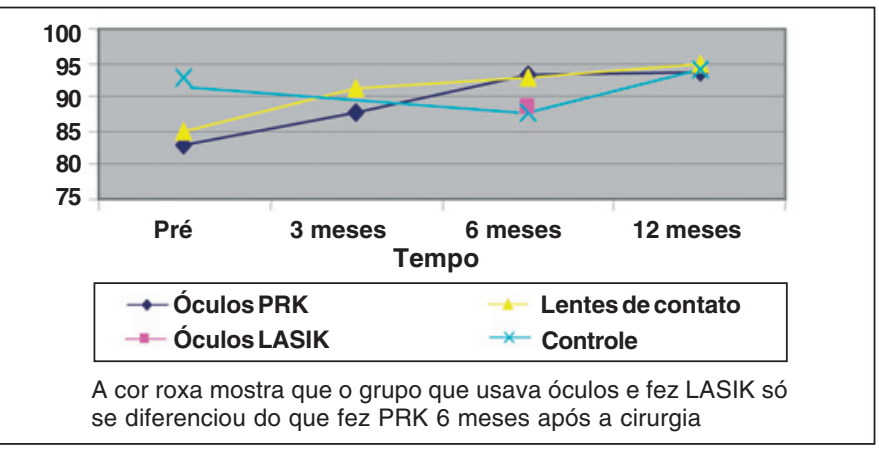

Gráfico 1 - Resultados do total do NEIVFQ dos grupos ao longo do ano

\begin{tabular}{|c|c|c|c|c|c|c|c|c|}
\hline & \multicolumn{2}{|c|}{ Pré } & \multicolumn{2}{|c|}{3 meses } & \multicolumn{2}{|c|}{6 meses } & \multicolumn{2}{|c|}{12 meses } \\
\hline & NEIVFQ & SRQ & NEIVFQ & SRQ & NEIVFQ & SRQ & NEIVFQ & SRQ \\
\hline Controle & 92,66 & 3,80 & & & 87,74 & 3,69 & 94,08 & 3,04 \\
\hline Óculos LASIK & 84,08 & 4,14 & 85,92 & $2,76^{*}$ & $88,35^{*}$ & 2,83 & 93,71 & 2,05 \\
\hline Óculos PRK & 82,67 & 4,14 & 89,56 & $2,76^{*}$ & $93,44^{*}$ & 2,90 & 95,36 & 2,05 \\
\hline Lente LASIK & 81,85 & 3,23 & 89,86 & 2,95 & 92,46 & 1,93 & 93,71 & 1,26 \\
\hline
\end{tabular}




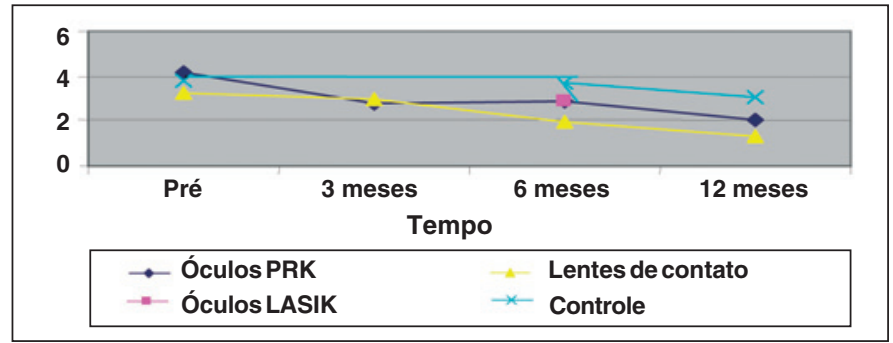

Gráfico 2 - Resultados do SRQ dos grupos ao longo do ano

\begin{tabular}{|c|c|c|c|c|c|}
\hline & \multicolumn{5}{|c|}{ Pacientes que usavam óculos } \\
\hline & Pré & 3 meses & 6 meses & LASIK & 12 meses \\
\hline Saúde geral & 65,27 & 63,85 & 71,73 & 62,50 & 72,79 \\
\hline Visão geral & 76,29 & 80,00 & 82,60 & 78,33 & 89,41 \\
\hline Sofrimento ocular & 80,78 & 85,75 & 93,44 & 88,35 & 89,33 \\
\hline Visão para perto & 86,65 & 88,25 & 96,12 & 89,58 & 96,93 \\
\hline Visão para longe & 78,04 & $89,55^{*}$ & 97,82 & 87,78 & 96,49 \\
\hline Atividade social & 95,37 & 99,03 & 100,00 & 99,30 & 99,75 \\
\hline Saúde mental & 72,96 & $82,80^{*}$ & 90,00 & 84,58 & 90,44 \\
\hline Dependência & 94,87 & 98,54 & 99,72 & 100,0 & 99,81 \\
\hline Desemp. papéis & 83,44 & $98,50^{*}$ & 99,45 & 97,91 & 99,81 \\
\hline Dirigir & 81,00 & 87,50 & 92,22 & 80,55 & 86,99 \\
\hline Visão para cores & 93,51 & 98,26 & 92,22 & 100,00 & 100,00 \\
\hline Visão periférica & 89,35 & 91,27 & $98,91^{*}$ & 91,66 & 100,00 \\
\hline
\end{tabular}

Tabela 5. Resultados dos quesitos do NEIVFQ para usuários de lentes de contato ao longo do ano

\begin{tabular}{|lcccc|} 
& \multicolumn{5}{c}{ Pacientes que usavam lentes de contato } \\
\cline { 2 - 5 } Saúde geral & Pré & 3 meses & $\mathbf{6}$ meses & 12 meses \\
Visão geral & 72,61 & 69,11 & 75,00 & 85,00 \\
Sofrimento ocular & 75,23 & 82,35 & 86,66 & 88,00 \\
Visão para perto & 89,19 & $92,64^{*}$ & 92,50 & 95,00 \\
Visão para longe & 78,57 & 93,87 & 94,72 & $96,66^{*}$ \\
Atividade social & 96,85 & 100,00 & 100,00 & 99,44 \\
Saúde mental & 76,90 & $86,76^{*}$ & 91,66 & 91,66 \\
Dependência & 94,34 & 98,90 & 100,00 & 99,58 \\
Desemp. papéis & 83,92 & $97,80^{*}$ & 99,00 & 99,58 \\
Dirigir & 82,41 & 85,70 & 86,59 & 87,81 \\
Visão para cores & 96,42 & 100,00 & 96,66 & 98,33 \\
Visão periférica & 84,52 & 98,52 & 95,00 & 100,00 \\
*= diferença estatística & significante & & \\
\hline
\end{tabular}

escolha e mais 14 perguntas opcionais. O objetivo do questionário é avaliar a qualidade de visão e de vida em doze diferentes áreas: saúde geral, visão geral, sofrimento ocular, visão para atividades para perto, visão para atividades para longe, visão para atividades sociais, conflitos emocionais, visão para desempenho de papéis, dependência, visão para dirigir automóveis, visão para cores e visão periférica. Para

\begin{tabular}{|lccc|}
\hline \multicolumn{3}{|c|}{$\begin{array}{c}\text { Tabela 6. Resultados dos quesitos do NEIVFQ do grupo controle ao lon- } \\
\text { go do ano } \\
\end{array}$} & \multicolumn{3}{c}{ Pacientes controle } \\
\cline { 2 - 4 } & Pré & $\mathbf{6}$ meses & $\mathbf{1 2}$ meses \\
Saúde geral & 76,00 & 72,82 & 75,00 \\
Visão geral & 89,60 & 89,56 & 86,95 \\
Sofrimento ocular & 89,50 & 86,95 & 93,47 \\
Visão para perto & 96,16 & 95,47 & 96,37 \\
Visão para longe & 94,17 & 94,92 & 95,79 \\
Atividade social & 98,66 & 98,55 & 99,63 \\
Saúde mental & 91,60 & 88,47 & 93,69 \\
Dependência & 99,50 & 98,91 & 99,45 \\
Desemp. papéis & 97,00 & 97,82 & 99,45 \\
Dirigir & 87,67 & 89,39 & 92,42 \\
Visão para cores & 97,00 & 98,91 & 100,00 \\
Visão periférica & 88,00 & 95,61 & 97,82 \\
\hline
\end{tabular}

cada item fez-se uma média aritmética. A soma das médias destes doze itens e a divisão por 12 resulta num escore total que, quanto mais próximo de 100 melhor será a qualidade de visão da pessoa avaliada.

O SRQ 20 foi elaborado em $(1980)^{(8)}$. Muitas de suas perguntas foram retiradas GHQ 12. O GHQ 12 não aborda questões de psicossomática, diferentamente do SRQ 20 que investiga dor de cabeça, falta de apetite e má digestão. O SRQ 20 foi desenvolvido para diagnosticar distúrbios psiquiátricos em cuidados primários ${ }^{(9)}$. O SRQ-20 é um questionário de rastreamento para transtornos psiquiátricos menores composto de 20 perguntas com o objetivo de avaliação do estado de saúde mental. O questionário é binomial: a resposta afirmativa indica presença de sintoma e a resposta negativa, a sua ausência. Neste questionário o paciente que responde sete ou mais perguntas positivamente é considerado com provável distúrbio psíquico.

No presente trabalho tanto a amostra do grupo de estudo como a do grupo controle eram semelhantes e pertenciam à classe média uma vez que o sistema único de saúde, bem como a grande maioria dos convênios médicos não cobria este tipo de cirurgia. Assim, evidentemente os resultados estão relacionados apenas a essa parcela da população, mas deve-se lembrar que é este o segmento da população que mais a procura.

Este estudo não teve por objetivo comparar PRK com LASIK no entanto isso aconteceu apenas no pós-operatório de 6 meses em que houve maior diferença nas ametropias entre os dois grupos.

Este estudo mostrou que nos pacientes que se submeteram à cirurgia a qualidade de vida e de visão melhoraram significantemente comparados com o pré e o estresse psicológico também diminuiu. O grupo controle não mostrou diferença ficando estável durante o ano. Consideramos que as respostas dos pacientes não foram influenciadas por já conhecerem as perguntas a partir da segunda aplicação dos questionários porque passavam-se no mínimo três meses entre uma aplicação e outra.

Os pacientes procuraram a cirurgia refrativa porque estavam 
insatisfeitos com o uso de óculos e lentes de contato. Alegavam que estes interferiam na estética e eram incompatíveis com atividades esportivas. No caso das lentes elas exigiam muitos cuidados na sua manipulação e manutenção. Ao grupo controle não foi perguntado o porquê não pretendiam fazer a cirurgia em um prazo de um ano. Simplesmente se pretendiam fazê-la ou não. Genericamente devia haver satisfação quanto ao uso dos óculos e lentes uma vez que estes não pretendiam realizá-la.

De acordo com a metodologia empregada os dois grupos apresentaram diferenças relacionadas à satisfação com uso dos óculos, necessidade dos mesmos para conseguir visão normal para longe e também quanto à saúde mental.

Os pacientes do grupo submetido à cirurgia, além de insatisfeitos quanto à visão e necessidade de uso de óculos, também referiam mais frustações e instabilidade além de menor autoconfiança. Também associaram o uso dos óculos ao menor sucesso afetivo e profissional, com pior qualidade de vida de acordo com o NEI-UFQ.

Seis meses e um ano após a cirurgia o grupo controle permaneceu estável e houve uma diferença significante entre os grupos de PRK e o de LASIK. Isso também aconteceu nos itens saúde geral, visão para longe e dirigir.

A comparação dos três grupos, ao longo de um ano, mostrou que os grupos de óculos e lentes de contato apresentaram melhora significante já três meses após a cirurgia nos seguintes itens: visão para longe, desempenho de papéis e SRQ 20, maior independência, além de menor ansiedade e estresse, semelhante a outros estudos ${ }^{(1,11)}$.

Houve nos dois grupos operados redução significante de sintomas sugestivos da doença comportamental um ano após a cirurgia. Foram evidentes nos dois grupos operados as melhorias significantes das condições visuais e de vida, corroborando o grande impacto psicológio da cirurgia refrativa.

$\mathrm{O}$ estresse dos pacientes foi notado antes da cirurgia pela preocupação quanto ao ato cirúrgico e o seu resultado. Houve melhora significante três meses após a cirurgia no item saúde mental, provavelmente por sentirem menor frustração, menor irritabilidade e maior autoconfiança bem como na visão geral e sofrimento ocular.

O grupo que usava óculos mostrou diferença significante também quanto à melhora da visão para perto um ano após a cirurgia apesar da cirurgia ter efeito apenas para a visão para longe. A eficácia das cirurgias para miopia na melhora da qualidade de vida, de visão mostrada neste estudo está de acordo com os trabalhos de outros autores e o objetivo da cirurgia, foi alcançado ${ }^{(1,5)}$.

\section{CONCLUSÕES}

O grupo de pacientes operado melhorou a qualidade de vida e diminuiu o estresse psicológico enquanto o grupo controle que não quis se submeter à cirurgia se manteve estável durante o estudo.

\section{ABSTRACT}

Purpose: To evaluate the quality of life, vision and stress before and after refractive surgery. Methods: This is a longitudinal, observational study, where 100 patients were evaluated; 54 espectacles wearers, 21 contact lens users before surgery and 25 controls wearers spectacles or contact lenses, who did not want to undergo refractive surgery during one year despite refractive error. The applied questionnaires were Self Reporting Questionnaires SRQ-20 of quality of life and vision and National Eye Institute Visual Function Questionnaire NEI VFQ-25 for the assessment of mental health. The intervention group answered the questionnaires before surgery, three, six and twelve months after follow-up and the control group answered the questionnaires at six and twelve months after the baseline. The questionnaires of the intervention group were applied by an independent person. Results: In the intervention group (54 spectacle wearers), 39 were treated by photorefractive excimer laser keratectomy (PRK) and 15 by laser in situ keratomileusis (LASIK), of the 21 contact lens users: 12 received photorefractive excimer laser keratectomy and nine were treated by laser in situ keratomileusis (LASIK). The control group remained stable during the study. After three months of follow-up the intervention group showed improvement in quality of life, vision and reduction of stress. After one year of follow-up the assessments of quality of life and mental health were similar to the control group. After three months there was a significant reduction of psychiatric symptomatology in the intervention group. Conclusion: Patients operated for correction of ametropia showed a significant improvement in quality of life and mental health assessments.

Keywords: Keratomileusis, laser in situ; Keratectomy, photorefractive, excimer laser; Refractive errors/surgery; Myopia/ surgery; Quality of life; Stress, psychological; Questionnaires

\section{REFERÊNCIAS}

1. Freitas C, Oliveiros BM, Marques E, Leite EB. Effect of photorefractive keratectomy on visual functioning and quality of life. J Refract Surg. 1995; 11(3 Suppl):S327-34.

2. Bourque LB, Rubenstein R, Cosand B, Waring GO $3^{\text {rd }}$, Moffitt S, Gelender H, et al. Psychosocial characteristics for candidates for the prospective evaluation of radial keratotomy (PERK) study. Arch Ophthalmol. 1984;102(8):1187-92.

3. Powers MK, Meyerowitz BE, Arrowsmith PN, Marks RG. Psychosocial finding in radial keratokomy patients two years after surgery. Ophthalmology. 1984;91(1):1193-8.

4. Bourque LB, Cosand BB, Drews C, Waring GO $3^{\text {rd }}$, Lynn M, Cartwright C. Reported satisfaction, fluctuation of vision and glare among patients one year after surgery in the Prospective Evaluation Of Radial Keratotomy (PERK) study. Arch Ophthalmol. 1986;104(3):356-63.

5. Kidd B, Stark C, McGhee CN. Screening for psychiatric distress and low self esteem in patients presenting for excimer laser surgery for myopia. J Refract Surg. 1997;13(1):40-4.

6. Rose K, Harper R, Tromans C, Waterman C, Goldberg D, Haggerty C, et al. Quality of life in myopia. Br J Ophthalmol. 2000;84(9):1031-4.

7. Lau J, Michon JJ, Chan WS, Ellwein LB. Visual acuity and quality of life outcomes in cataract surgery in in Hong Kong. Br J Ophthalmol. 2002;86(1):12-7.

8. Harding TW, de Arango MV, Baltazar J, Climent CE, Ibrahim HH, Ladrido- 
Ignacio L, et al. Mental disorders in primary health care: a study for their frequency and diagnosis in four developing countries. Psychol Med. 1980; 10(2):231-41.

9. Goldberg DP, Cooper B, Eastwood MR, Kedward HB, Shepherd M. A standardized psychiatric intervew for use in community surveys. Br J Prev Soc Med. 1970;24(1):18-23.

10. Mari JJ, Williams P. A comparisom of the validity of two psychiatric screening questionnaires (GHQ - 12 and SRQ - 20) in Brasil, using Relating Operating Characteristic (ROC) analysis. Psychol Med. 1985;15(3):651-9.

11. Lima MS, Beria JU, Tomasi E, Conceição AT, Mari JJ. Stressful life events and minor psychiatric disorders: an estimate of the population attributable fraction in the Brazilian community - based study. Int J Psychiatry Med. 1996; 26(2):211-22.

12. Mangione CM, Lee PP, Gutierrez PR, Spritzer K, Berry S, Hays RD. National Eye Institute Visual Function Questionnaire Field Test Investigators. Development of the 25-item National Eye Institute Visual Function Questionnaire. Arch Ophthalmol. 2001;119(7):1050-8.

13. Holander M, Wolfe DA. Nonparametric Statistical Methods. New York: Willey; c1975. 503p.

14. Siegal S. Estatística não paramétrica para as ciências do comportamento. São Paulo, Mc Grow - Hill; c1975. 350p.

\title{
VIII Curso Certificado de Treinamento Técnico e Científico em Banco de Olhos no Brasil - APABO
}

\section{0 e 26 de julho de 2008 São Paulo - SP}

Idioma Oficial: Português (para os representantes dos Bancos de Olhos brasileiros)

\author{
INFORMAÇÕES \\ APABO (Escritório Brasil) \\ Tel/Fax: (35) 3421.2186 (exclusivo para o Brasil) \\ E-mail: apabo@uninet.com.br \\ Site: http://www.apabo.com.br
}

